\title{
Analysis, Modeling and Compensation of Dynamic Imbalance Error for a Magnetically Suspended Sensitive Gyroscope
}

\author{
Xin Chaojun ${ }^{1}$, Cai Yuanwen ${ }^{1}$, Ren Yuan ${ }^{1 *}$, Fan Yahong ${ }^{2}, X$ Guofeng ${ }^{1}$, and Lei $\mathrm{Xu}^{3}$ \\ ${ }^{1}$ Equipment Academy, Beijing, China \\ ${ }^{2}$ Beijing Institute of Control Engineering, Beijing, China \\ ${ }^{3}$ Jiuquan Satellite Launch Center, Gansu, China
}

(Received 16 August 2016, Received in final form 13 October 2016, Accepted 17 October 2016)

\begin{abstract}
Magnetically suspended sensitive gyroscopes (MSSGs) provide an interesting alternative for achieving precious attitude angular measurement. To effectively reduce the measurement error caused by dynamic imbalance, this paper proposes a novel compensation method based on analysis and modeling of the error for a MSSG. Firstly, the angular velocity measurement principle of the MSSG is described. Then the analytical model of dynamic imbalance error has been established by solving the complex coefficient differential dynamic equations of the rotor. The generation mechanism and changing regularity of the dynamic imbalance error have been revealed. Next, a compensation method is designed to compensate the dynamic imbalance error and improve the measurement accuracy of the MSSG. The common issues caused by dynamic imbalance can be effectively resolved by the proposed method in gyroscopes with a levitating rotor. Comparative simulation results before and after compensation have verified the effectiveness and superiority of the proposed compensation method.
\end{abstract}

Keywords : Magnetically suspended sensitive gyroscope (MSSG), dynamic imbalance, angular velocity, error compensation method, measurement accuracy

\section{Introduction}

With the advantages of frictionless, no wear, without lubricating, low consuming and long life, the magnetically suspended rotor has been applied in many space missions as ideal attitude control system actuators with high accuracy [1-4]. In recent years, researchers have begun to develop inertial sensors based on magnetically suspended rotor, named magnetically suspended sensitive gyroscope (MSSG) [5-7]. A levitated rotor is supported by the magnetic pulls produced by the magnetic bearings in the stator, rotating at high angular velocity. Based on the law of angular momentum, the attitude angles or angular velocities would be measured by a MSSG.

Due to the material inhomogeneity, manufacture precision, assembly error and other aspects of factors, the mass center of magnetically suspended rotor may deflects from its geometry center. This is called a static imbalance, since gravity turns the rotor even when the motor does

CThe Korean Magnetics Society. All rights reserved.

*Corresponding author: Tel: +86-10-18610226728

Fax: +86-10-6636-4198, e-mail: jingninan@126.com not. Or, the principle axis of inertia of the rotor does not coincide with its rotation axis. This is called a dynamic imbalance, since a torque is exerted on the rotor when it spins, but no imbalance is detected when the rotor is stationary [8]. These imperfections lead to additional disturbance torques acting on the spinning rotor. The amplitude of the disturbance torques is proportional to the square of the angular velocity. The disturbance torques will cause drift and affect the measurement accuracy of a MSSG.

Researchers have worked extensively on modeling and compensation of gyroscope output [9-12], improving the performance of MSSGs to a certain degree. Being a newly developed gyroscope, the affect of the dynamic imbalance to measurement accuracy have not been studied thoroughly. However, much work has been done in compensating the dynamics imbalance of the magnetically suspended rotors, which can provide meaningful referencing value to the study of MSSG.

Since the magnetic bearing possesses the feature of active controlling, many researchers compensate the dynamics imbalanced of the magnetically suspended rotor by adaptive control methods. The current regulation control can 
be achieved by an observer-based control, and eliminates the stationary alternating signals caused by the dynamic imbalance in the control currents. However, because the actual structure of rotor is asymmetry axially, approximately $30 \%$ residual oscillating component still exists [13]. To eliminate the oscillating component in the control current, Takeshi Mizuno et al. applied the frequencytracking servocompensator in the hybrid suspended gyroscope [14]. Experiment shows that the component synchronized with the rotor spin is eliminated completely. Schuhmann et al. [15] implemented a Kalman filter and an optimal state feedback regulator to improve rotor positioning accuracy. Based on the estimation of disturbance forces, the forces caused by the dynamic imbalance of the magnetically levitated rotor are compensated credibly. Fan Yihua et al. [16] used a model-based force estimator with an adaptive tuner for a magnetically suspended rotor to eliminate the unbalancing vibration. By means of adding the estimator to an observer, the vibration associated with dynamic imbalance is suppressed over all of the operational range. Queiroz proposed an active feedback method for identifying the unknowing imbalance parameters of a rotating rotor with time-varying rotation velocity via a pair of active radial control forces [17]. Cui Peiling investigated the method for adaptive dynamic imbalance vibration control of the variable-speed rotor [18]. Based on the feedforward compensation, a parameter modifier of power amplifier with control switches is added to eliminate the unbalancing vibration, and the method has a high compensation precision.

The other adopted methods includes notch filters, LMS, etc [11]. The above researches compensate the dynamic imbalance of magnetically suspended rotors to a certain extent. By making the rotor rotate around the geometric axis, the rotation accuracy has achieved great improvement.

However, these researches focus on the vibration suppressing which caused by the static and dynamic mass imbalance, regarding the spinning rotor systems as actuators exclusively. On one side, extra apparatuses are added to the control system, on the other side, complicated controlling algorithms increase the calculation time, and new problems have been introduced. There are few theoretical studies available for dynamic imbalance analysis while taking magnetically suspended rotor system as an inertial sensor. Based on the newly developed MSSG, this paper introduced the error caused by dynamic imbalance of the rotor. Aiming to compensate the error in gyro output, thus improve the measurement accuracy of a MSSG, the analytical expression of the dynamic imbalance error has been deduced, and the characteristics of dynamic imbalance error have been analyzed deeply. Then an universal method to compensate the dynamic imbalance error for gyroscopes with a levitating rotor has been proposed.

The remainder of this paper is organized as follows. In Section 2, we described the fundamental structure and the measurement principle of the MSSG. Next, the theoretical model of dynamic imbalance error is derived, and its changing regularity has been analyzed. Meanwhile the model of the measuring error caused by the dynamic imbalance has been built in Section 3. In section 4, the compensation method for dynamic imbalance error has been brought forward, and section 5 conducts the comparative simulations to testify the measurement performance with the proposed compensation method. Follow on, Section 6 concludes this paper.

\section{Composition and Measurement Principle of a MSSG}

\subsection{Composition of a MSSG}

A magnetically suspended sensitive gyroscope system has been built for testing in the Institute of Advanced Inertial Measuring and Control, Equipment Academy. Figure 1 shows the main configuration of the MSSG. The MSSG studied in this paper consists of rotor, stator, high speed motor, torquer, a gyro house and displacement sensors. The rotor of the MSSG is a type of spherical in structure. Radiuses of rotor in axial and radial are different, while the radius is $r_{1}$ in radial and $r_{2}$ in axial. The rotor is made of magnetic material in whole. When the rotor is suspended in the center of the rotor housing, the bearing gap between the rotor and the inner surface of the stator magnetic poles is $0.35 \mathrm{~mm}$. The rotor is levitating in the center of the stator cavity by magnetic pull caused by the interaction of the electromagnetic stator and the rotor. The rotation of the rotor is driven by a hollow cup permanent magnet brushless DC motor, whose coils are cured on the frame of the cup-chapped motor stator. The motor magnets are installed in the rotor of the MSSG with four pole structure [9]. When the magnetic pole envelope surfaces (MPESs) of stator and the rotor are ideal spherical surfaces, the magnetic forces over the entire surface of the rotor will pass through its geometric center and no torques are produced. The torquer is a Lorentz-type magnetic bearing, which is used to control the tilting of the rotor. The Lorentz-type magnetic bearing consists of permanent magnets, Lorentz coils, frame, sensors and associated peripherals. Two ring-type permanent magnets are fixed in the circumferential slot in the rim of the MSSG rotor to produce a circumferentially uniform magnetic field. Four Lorentz coils are fixed symmetrically in the $\mathrm{x}$ - and $\mathrm{y}$-axis 


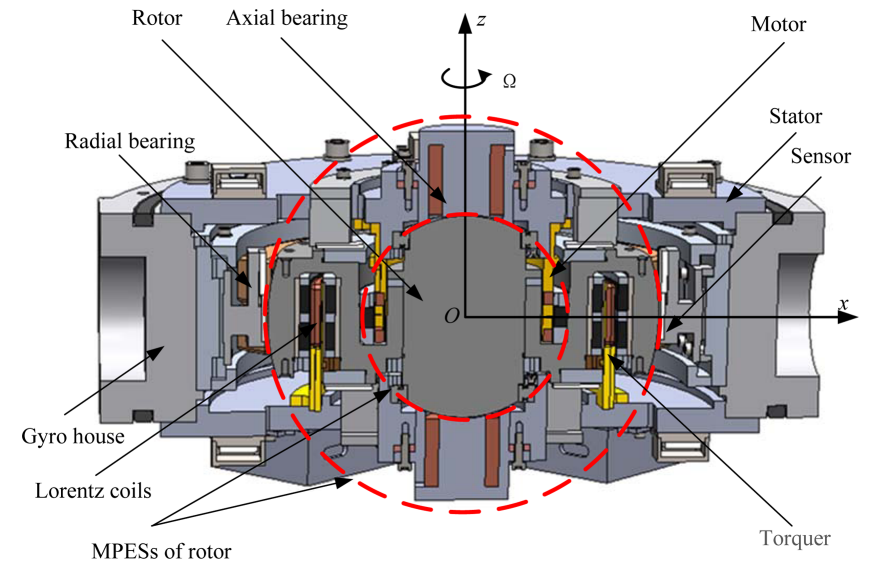

Fig. 1. (Color online) Configuration of the MSSG.

on the frame, and the coils on the frame are located in the circumferentially magnetic field. When the control current was applied to one of the coils by the control system, Lorentz force will generate according to Ampere's law, which will produce a deflection control moment to compel the rotor rotate.

\subsection{Principle of measurement}

When the gyro-carrier is static, the rotor is levitating in the center of the gyro house, spinning about $z$-axis at angular rate $\Omega$, as is shown in Fig. 1. However, if the gyro-carrier fixed with the gyro house comes into an offaxis attitude angular rate $\omega_{i b}$ related to the inertial space, the attitude of levitating rotor would remain the same dues to the gyroscopic inertia of the rotor. The displacement sensors would pick up the position changes of the rotor related to gyro house, and transmit the signals to control system. Control current will be applied to the Lorentz coils of the torquer by the control system according to the signals, compelling the rotor promptly rotate back to its equilibrium position at angular rate $\omega_{i r}$ related to the inertial space. The control bandwidth of the torquer and the measurement bandwidth of the displacement sensors are much higher than the attitude change frequency of the gyro-carrier, so within the control bandwidth, the rotor would always track the attitude angular rate of the gyro-carrier in $x$ - and $y$-axes, stabilizing the rotor at the predesigned position.

Attitude angular rate of the gyro-carrier can be described as

$$
\omega_{i b}=\omega_{i r}-\omega_{r b}
$$

where $\omega_{r b}$ is the deflection angular velocity of rotor related to the gyro house.

By using Euler dynamic equati ons, the equivalent moment acting on the rotor in $x$ - and $y$-directions as the gyro-carrier rotating can be obtained,

$$
\left\{\begin{array}{c}
P_{d x}=J_{z} \Omega \omega_{i b y}+J_{r} \dot{\omega}_{i b x} \\
P_{d y}=-J_{z} \Omega \omega_{i b x}+J_{r} \dot{\omega}_{i b y}
\end{array}\right.
$$

where $J_{z}$ is the polar moment of inertia of the rotor, and $J_{r}$ is the equator moment of inertia of the rotor, $\omega_{i b x}$ and $\omega_{i b y}$ is the attitude angular rate of gyro-carrier around $x$ - and $y$ axes, respectively. $P_{d x}$ and $P_{d y}$ are the deflection control moments produced by the torquer around $x$ - and $y$-axes.

When the angular acceleration of the gyro-carrier is small, the above formula can be turned into gyroscopic effect precession equation since $J_{z} \Omega \gg J_{r}$, and the attitude angular rate of the gyro-carrier can be formulated as

$$
\left\{\begin{array}{c}
\omega_{i b y}=\frac{P_{d x}}{J_{z} \Omega} \\
\omega_{i b x}=-\frac{P_{d y}}{J_{z} \Omega}
\end{array}\right.
$$

Ideally, the structure of the MSSG has determined the resultant moment acting on the levitating rotor generated from only the deflection control moment $P_{d x}, P_{d y}$, produced by the torquer. Hence, the attitude angular rate of the gyro-carrier can be acquired indirectly by means of measuring the control currents in the Lorentz coils of MSSG torque.

\section{Dynamic Imbalance Error Analysis and Modeling}

\subsection{Analysis of dynamic imbalance in a MSSG}

Figure 2 shows the diagram of the dynamic imbalance of the magnetically suspended rotor. The rotation axis is

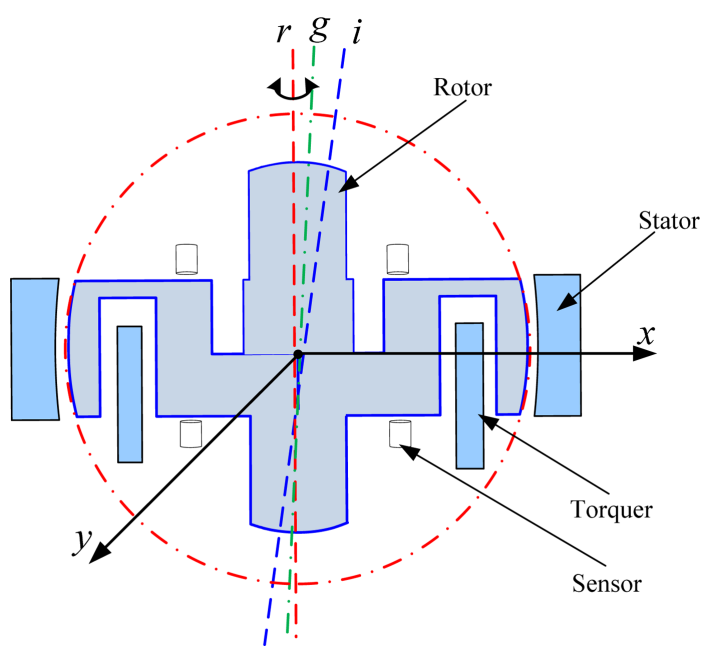

Fig. 2. (Color online) Dynamic imbalance diagram of MSSG. 


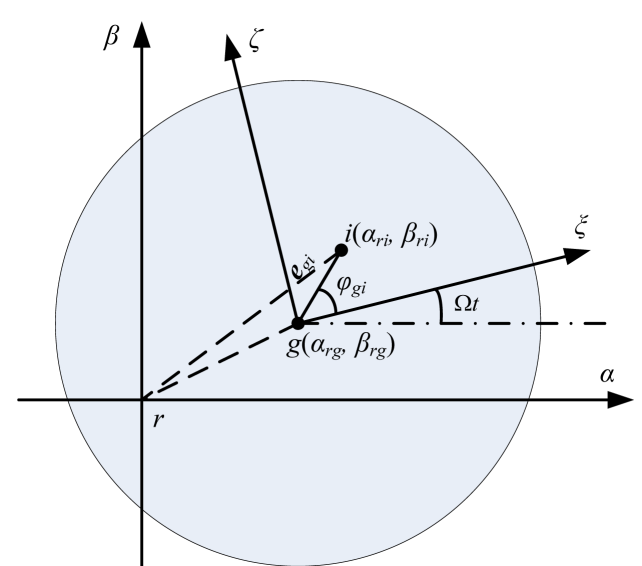

Fig. 3. (Color online) Relative angular position of each axis in MSSG

labeled as $r$, the principle axis of inertia and geometric axis are labeled as $i$ and $g$, respectively. Because of the existence of the dynamic imbalance, the principle axis of inertia $i$ does not coincide with the rotation axis.

Figure 3 is the diagram of relative angular position of each axis. In Fig. $3, r(\alpha, \beta)$ is the static angular coordinate system, $g(\xi, \zeta)$ is the rotating angular coordinate system attached to the rotor. The coordinate of the geometric axis in $r(\alpha, \beta)$ is $\left(\alpha_{r g}, \beta_{r g}\right)$, and that of principle axis of inertia is $\left(\alpha_{r i}, \beta_{r i}\right)$. The modulus of the principle axis of inertia is $e_{g i}$, and its phase is $\varphi_{g i}$ in $g(\xi, \zeta)$.

According to Euler dynamic equations, the dynamic equation of the rotor is then found to be

$$
\left\{\begin{array}{l}
J_{r} \ddot{\beta}_{r i}-J_{z} \Omega \dot{\alpha}_{r i}=P_{y} \\
J_{r} \ddot{\alpha}_{r i}+J_{z} \Omega \dot{\beta}_{r i}=P_{x}
\end{array}\right.
$$

where $P_{x}, P_{y}$ is the electromagnetic torques produced by the torquer in $\alpha$ and $\beta$ directions, respectively. The tilted angles measured by the sensors can be described as

$$
\left\{\begin{array}{l}
\alpha_{r g}=\alpha_{r i}-e_{g i} \cos \left(\Omega t+\varphi_{g i}\right) \\
\beta_{r g}=\beta_{r i}-e_{g i} \sin \left(\Omega t+\varphi_{g i}\right)
\end{array}\right.
$$

Substituting Equation (5) into (4), the equivalent dynamic equation becomes

$$
\left\{\begin{array}{l}
J_{r} \ddot{\beta}_{r g}-J_{z} \Omega \dot{\alpha}_{r g}-J_{r} \Omega^{2} e_{g i} \sin \left(\Omega t+\varphi_{g i}\right)+J_{z} \Omega^{2} e_{g i} \cos \left(\Omega t+\varphi_{g i}\right)=P_{y} \\
J_{r} \ddot{\alpha}_{r g}+J_{z} \Omega \dot{\beta}_{r g}-J_{r} \Omega^{2} e_{g i} \cos \left(\Omega t+\varphi_{g i}\right)-J_{z} \Omega^{2} e_{g i} \sin \left(\Omega t+\varphi_{g i}\right)=P_{x}
\end{array}\right.
$$

Equation (6) has divided the torques into four parts, the first two items on the left side of the formula are the centrifugal moment and gyroscopic moment produced by the non-coincidence of geometric axis and rotation axis, and the last two parts are the centrifugal moment and gyroscopic moment produced by the non-coincidence of principle axis of inertia and geometric axis.

Equation (6) can be represent in complex formation as

$$
J_{r} \ddot{\gamma}-J_{z} \Omega \dot{\gamma} \cdot j-\left(J_{r}-J_{z}\right) \mathbf{e}_{g i} \Omega^{2} e^{j \Omega t}-\mathbf{P}=0
$$

where $\gamma=\alpha_{r g}+\beta_{r g} \times j, \mathbf{P}=P_{y}+P_{x} \times j, j$ is the imaginary unit. And due to the structure of Lorentz force type magnetic bearing for the MSSG torquer, the electromagnetic torques acting on the rotor can be expressed as

$$
\mathbf{P}=-K \gamma-D \dot{\gamma}
$$

where $K$ is the stiffness coefficient, and $D$ is the damping coefficient of the torquer.

Substituting Equation (8) into (7), then we can get

$$
\ddot{\gamma}-\frac{J_{z}}{J_{r}} \Omega \dot{\gamma} \cdot j-\frac{\left(J_{r}-J_{z}\right)}{J_{r}} \mathbf{e}_{g i} \Omega^{2} e^{j \Omega t}+k^{2} \gamma+2 n k \dot{\gamma}=0
$$

where $k=\sqrt{K / J_{r}}$, and $n=D / 2 k J_{r}$.

Then Equation (9) can be transformed to a complex coefficient differential equation as,

$$
\ddot{\gamma}+\left(2 n k-\frac{J_{z}}{J_{r}} \Omega \cdot j\right) \dot{\gamma}+k^{2} \gamma=\frac{\left(J_{r}-J_{z}\right)}{J_{r}} \mathbf{e}_{g i} \Omega^{2} e^{j \Omega t}
$$

Obviously, Equation (10) is a typical equation of forced vibration. If there is no dynamic imbalance of the rotor, the rotor will be in the state of free vibration. And the general solution of the above equation can be calculated as follows,

$$
y=c_{1} e^{\lambda_{1} t}+c_{2} e^{\lambda_{2} t}
$$

where $c_{1}, c_{2}$ are arbitrary real constants. And $\lambda_{1}, \lambda_{2}$ can be represented as,

$$
\begin{aligned}
& \lambda_{1}=-n k-\frac{\sqrt{-\Delta}}{2} \sqrt{\sqrt{\lambda_{0}^{2}+1}+\lambda_{0}}-\frac{1}{2}\left(-A \Omega-\frac{\sqrt{-\Delta}}{\sqrt{\sqrt{\lambda_{0}^{2}+1}+\lambda_{0}}} j\right) \\
& \lambda_{2}=-n k+\frac{\sqrt{-\Delta}}{2} \sqrt{\sqrt{\lambda_{0}^{2}+1}+\lambda_{0}}-\frac{1}{2}\left(-A \Omega+\frac{\sqrt{-\Delta}}{\sqrt{\sqrt{\lambda_{0}^{2}+1}+\lambda_{0}}} j\right)
\end{aligned}
$$

where, $\lambda_{0}=-\left(4 k^{2}+A^{2} \Omega^{2}-4 n^{2} k^{2}\right) / 4 n k A \Omega, \Delta=-2 n k A \Omega, A$ $=J_{z} / J_{r}$. When the value of $n, k$ satisfy the following conditions, the free vibration would attenuate off soon and need not to be considered.

$$
\begin{aligned}
& -n k-\frac{\sqrt{-\Delta}}{2} \sqrt{\sqrt{\lambda_{0}^{2}+1}+\lambda_{0}}+\frac{1}{2} A \Omega<0 \\
& -n k+\frac{\sqrt{-\Delta}}{2} \sqrt{\sqrt{\lambda_{0}^{2}+1}+\lambda_{0}}+\frac{1}{2} A \Omega<0
\end{aligned}
$$

The non-homogeneous particular solution which corre- 
sponds to the steady state motion of the rotor can be indicated as,

$$
z=\frac{\frac{\left(J_{r}-J_{z}\right)}{J_{r}} \mathbf{e}_{g i} \Omega^{2}}{\left(2 n k \Omega i-\frac{J_{r}-J_{z}}{J_{r}} \Omega^{2}\right)+k^{2}} e^{j \Omega t}=\sqrt{M^{2}+N^{2}} e^{j(\Omega t+v)}
$$

where,

$$
\begin{aligned}
& M=\frac{\left(B \mathbf{e}_{g i} \Omega^{2} k^{2}-B^{2} \mathbf{e}_{g i} \Omega^{4}\right)}{\left(k^{2}-B \Omega^{2}\right)^{2}-(2 n k \Omega)^{2}} \\
& N=-\frac{\left(2 n k B \mathbf{e}_{g i} \Omega^{3}\right)}{\left(k^{2}-B \Omega^{2}\right)^{2}-(2 n k \Omega)^{2}} \\
& \nu=\arctan (N / M) \\
& B=\frac{\left(J_{r}-J_{z}\right)}{J_{r}}
\end{aligned}
$$

The steady state motion that the solution represents is that the deflected angle of the rotor geometric axis rotates at the speed of $\Omega$, but its phase lags behind the rotation of principle axis of inertia. Modulus and phase difference of the deflected angle are the functions of $\Omega, k$, and $n$.

By introducing the dimensionless parameter $s=\Omega / k$, modulus and phase difference of the deflected angle can be written as

$$
\begin{aligned}
& \frac{\sqrt{M^{2}+N^{2}}}{\mathbf{e}_{g i}}=\frac{B s^{2} \sqrt{1-2 B s^{2}+4 n^{2} s^{2}+B^{2} s^{4}}}{\left(1-B s^{2}\right)^{2}-(2 n s)^{2}} \\
& v=\arctan \left(\frac{2 n s}{1-B s^{2}}\right)
\end{aligned}
$$

The Equation (16) in the $\left(s-\sqrt{M^{2}+N^{2}} / \mathbf{e}_{g i}\right)$ coordinate plane is a family of amplitude-frequency curve, parametering for $n$, as is shown in Fig. 4. The angular velocity corresponding to the peak of deflected angle modulus is called critical angular velocity $\omega_{c r}$. When the actual speed of the rotor approaches critical angular velocity, the MSSG becomes resonance, and the rotor generate the largest deflected angle. Let the derivative of $\sqrt{M^{2}+N^{2}} / \mathbf{e}_{g i}$ with respect to $s$ to be 0 , and then we can solve the critical angular velocity and corresponding peak of deflected angle.

According to Fig. 4, we can discover that along with the increasing of damping coefficient, the critical angular velocity becomes lower and the peak of deflected angle increases. Figure 5 shows the family of phase-frequency curve, from which we can conclude that with the increasing of $n$, the phase difference of the deflected angle becomes larger, while along with the increasing of $\Omega$ the phase difference grows before $\Omega=k$ firstly, and then reduces

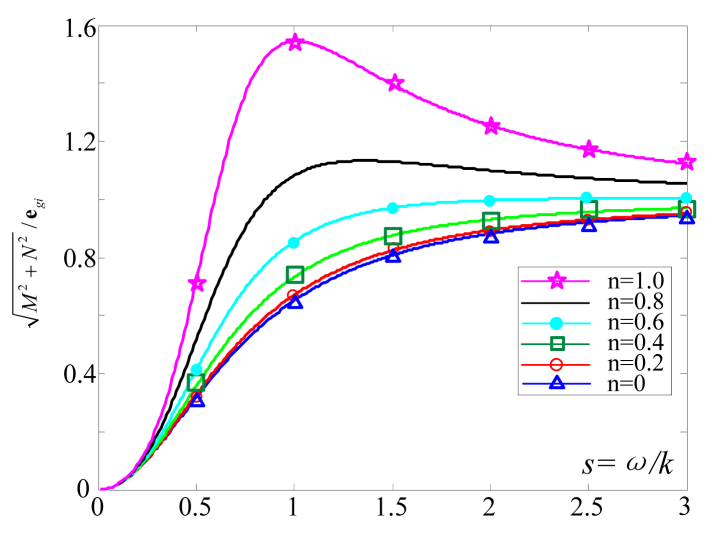

Fig. 4. (Color online) Amplitude-frequency curves of dynamic imbalance error.

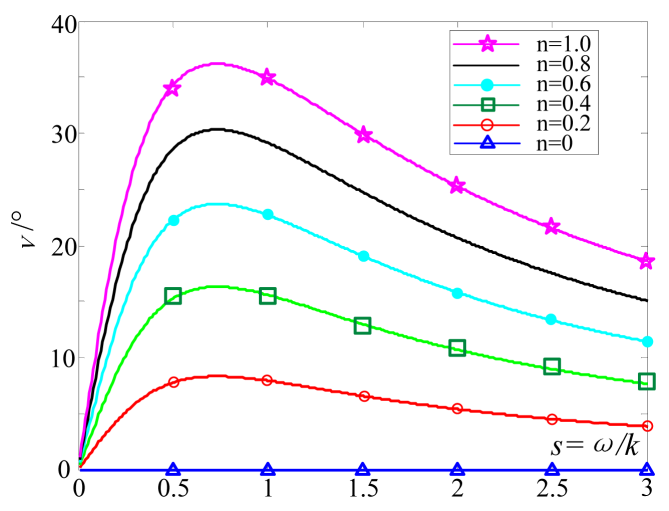

Fig. 5. (Color online) Phase-frequency curves of dynamic imbalance error.

gradually.

\subsection{Modeling of dynamic imbalance error in MSSG}

According to Equation (14), we can obtain the coordinate of the geometric axis in angular coordinate system $r(\alpha, \beta)$

$$
\left\{\begin{array}{l}
\alpha_{r g}=\sqrt{M^{2}+N^{2}} \cos (\Omega t+v) \\
\beta_{r g}=\sqrt{M^{2}+N^{2}} \sin (\Omega t+v)
\end{array}\right.
$$

Since $\alpha_{r g}, \beta_{r g}$ represent the titled angles of the rotor about $x$ - and $y$-axis respectively, the additional velocity of titled angle which is generated by the dynamic imbalance can be calculated by taking the derivative of the above equation,

$$
\left\{\begin{array}{l}
\Delta \omega_{x}=-\Omega \sqrt{M^{2}+N^{2}} \sin (\Omega t+v) \\
\Delta \omega_{y}=\Omega \sqrt{M^{2}+N^{2}} \cos (\Omega t+v)
\end{array}\right.
$$

Figure 6 helps to illustrate the mechanics leading to the generation of this additional velocity of deflected angle. If 


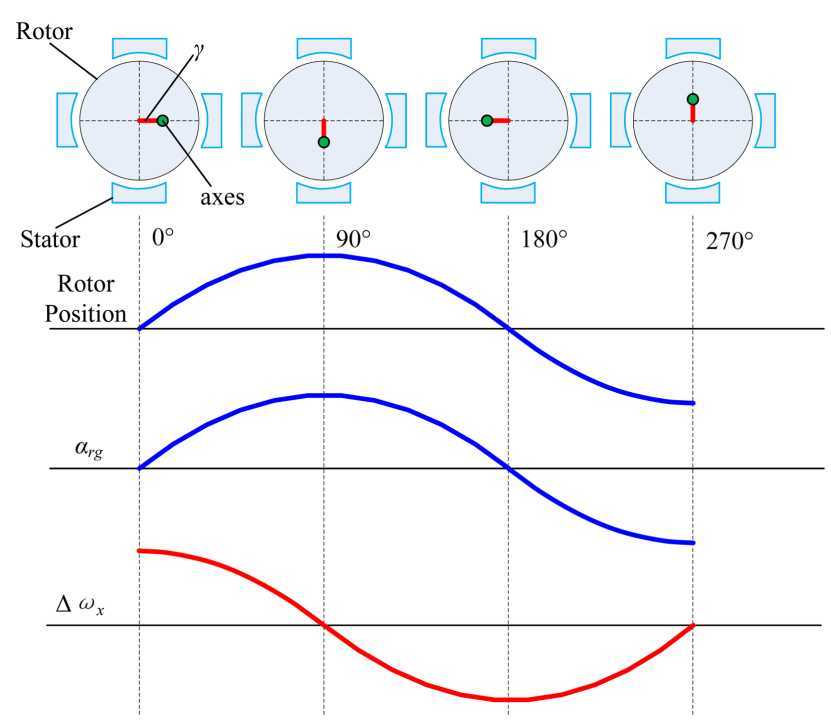

Fig. 6. (Color online) Explanation of dynamic imbalance error in a MSSG.

one considers the rotor of the MSSG rotating through $360^{\circ}$, at its start point, the deflected angle of geometric axis of rotor around $x$-axis $\alpha_{r g}$ is 0 . When the rotor rotates a quarter turn, $\alpha_{r g}$ becomes its maximum. After half revolution, the deflected angle returns to 0 . At the three quarter turn, $\alpha_{r g}$ becomes its maximum again, but in the opposite direction. Over all the course of a full turn, the deflected angle varies continually between 0 and its maximum, and a corresponding velocity of deflected angle exists. The velocity of deflected angle around $y$-axis can be analyzed in the same way.

As is seen from Equations (1), (3) and (18), due to the existence of the dynamic imbalance, there is an angular velocity error $\Delta \omega$ between the nominal angular velocity $\omega_{i b}$ and the true value of angular velocity $\hat{\omega}_{i b}$

$$
\Delta \omega=\hat{\omega}_{i b}-\omega_{i b} .
$$

\section{Method of Compensation}

To effectively increased the measurement accuracy of the MSSG, we have to reduce the angular velocity error caused by the dynamic imbalance of the rotor. The method of compensation the dynamic imbalance error for the MSSG can be obtained in the basis of the above analysis. And the compensation flow of the angular velocity error caused by dynamic imbalance is shown in Fig. 7.

(1) The control current $I$ of the Lorentz-type magnetic bearing torquer and spinning velocity $\Omega$ of the rotor are collected;

(2) Making use of Equation (3) to acquire the nominal

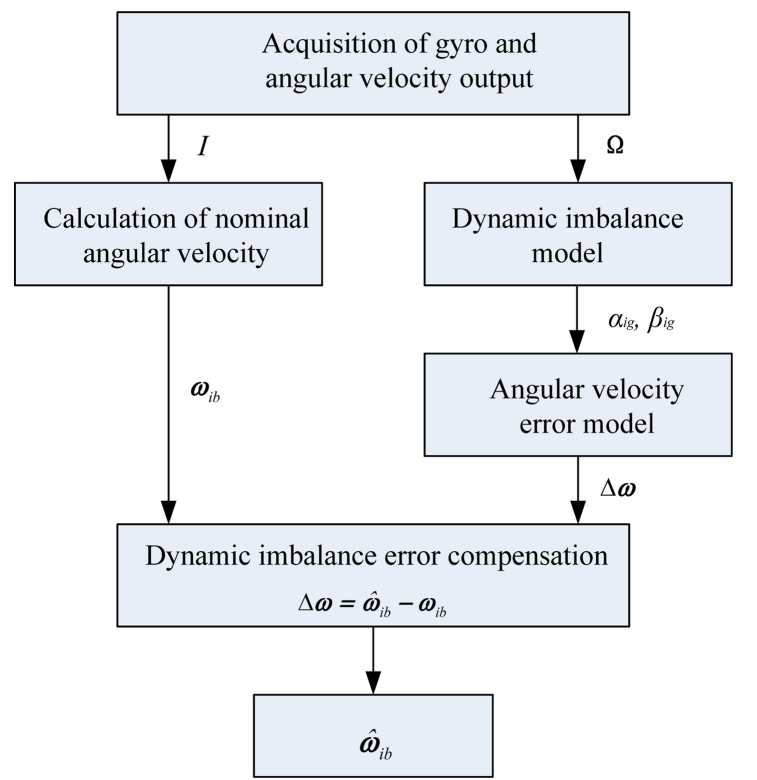

Fig. 7. (Color online) The flow of dynamic imbalance error compensation.

angular velocity $\boldsymbol{\omega}_{i b}$;

(3) The rotor spinning velocity is sent to dynamic imbalance error model Equation (14), which figures out the angular velocity error $\Delta \omega$ according to Equation (19);

(4) Equation (20) is utilized to calculate the angular velocity, and estimate the ture value of angular velocity precisely.

The experiment data collected in this paper include the output change of MSSG which caused by changes in titled angle of the rotor, and can be expressed as change of angular velocity error $\Delta \omega$. Therefore the model proposed in this paper contains the error caused by dynamic imbalance. And the proposed method corresponding to the model can compensate the error in the whole spinning velocity range.

\section{Simulation Results}

In this section, we conduct the simulations of the proposed method to verify the satisfactory compensation effect under the rated rotor speed $15000 \mathrm{r} / \mathrm{min}$. The

Table 1. System parameters of the MSSG.

\begin{tabular}{cccc}
\hline \hline $\begin{array}{c}\text { Parameter } \\
\text { (unit) }\end{array}$ & $\begin{array}{c}\text { Numerical } \\
\text { value }\end{array}$ & $\begin{array}{c}\text { Parameter } \\
\text { (unit) }\end{array}$ & $\begin{array}{c}\text { Numerical } \\
\text { value }\end{array}$ \\
\hline$m(\mathrm{~kg})$ & 4.7 & $J_{r}\left(\mathrm{~kg} \cdot \mathrm{m}^{2}\right)$ & 0.0034 \\
$\mathrm{k}$ & 1000 & $J_{z}\left(\mathrm{~kg} \cdot \mathrm{m}^{2}\right)$ & 0.0052 \\
$\mathrm{n}$ & 0.6 & $r_{1}(\mathrm{~m})$ & 0.07865 \\
$\mathbf{e}_{g i}\left({ }^{\circ}\right)$ & 0.1 & $r_{2}(\mathrm{~m})$ & 0.041 \\
\hline
\end{tabular}




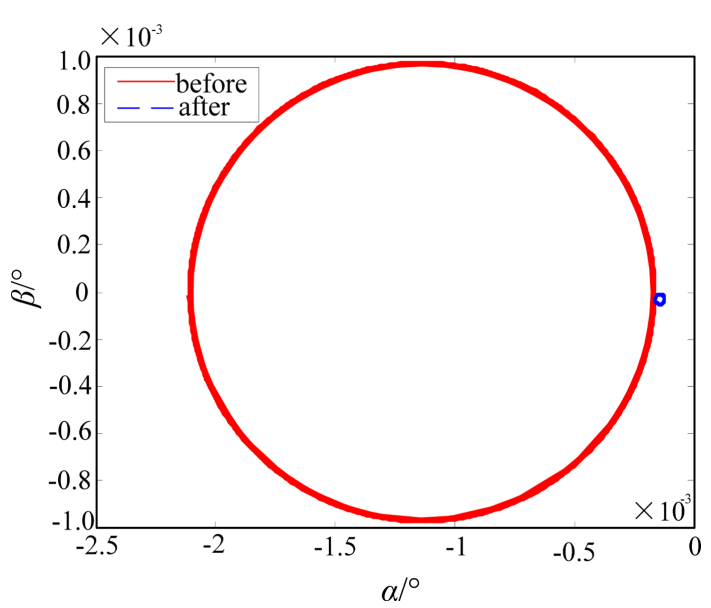

Fig. 8. (Color online) Orbit of the titled angles before and after compensation.

system parameters of the MSSG are shown in Table 1.

The orbits of the geometric axis tilted angles are shown in Fig. 8. The real (red) line delegates the orbit of tilted angles without dynamic imbalance compensation, and dotted (blue) line delegates the orbit of tilted angles after compensation, using the paper proposed method.

As is shown in Fig. 8, the orbit of the geometric axis tilted angles becomes smaller by a factor of 17 approximately after the compensation is carried out. Before compensation, the maximum of $\alpha$ is about $-2.2 \times 10^{-3 \circ}$, and after the compensation method has been carried out, the maximum of $\alpha$ becomes $-1.3 \times 10^{-40}$. Simultaneous, the maximum of $\beta$ diminishes from $9.4 \times 10^{-4 \circ}$ to $5.2 \times 10^{-50}$. These result shows that the compensation method is quite effective, and therefore makes great contribution for improving the measurement accuracy of the MSSG.

When there is an attitude angular rate of the gyro-carrier related to the inertial space, the measurement results will suffer a considering measuring error caused by the dynamic

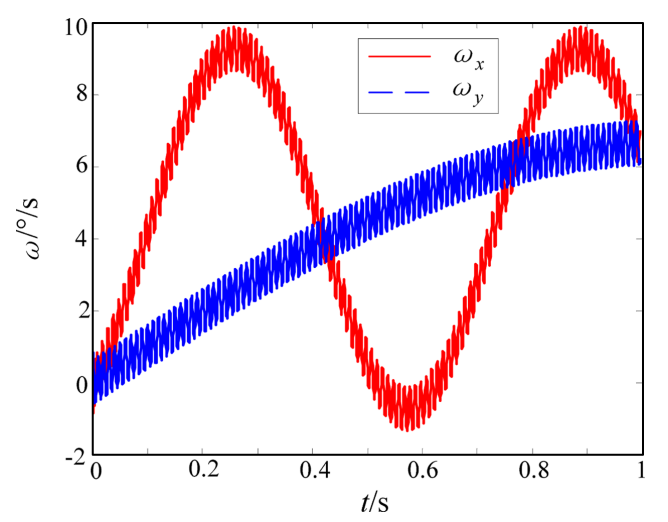

Fig. 9. (Color online) Measurement result of MSSG before compensation.

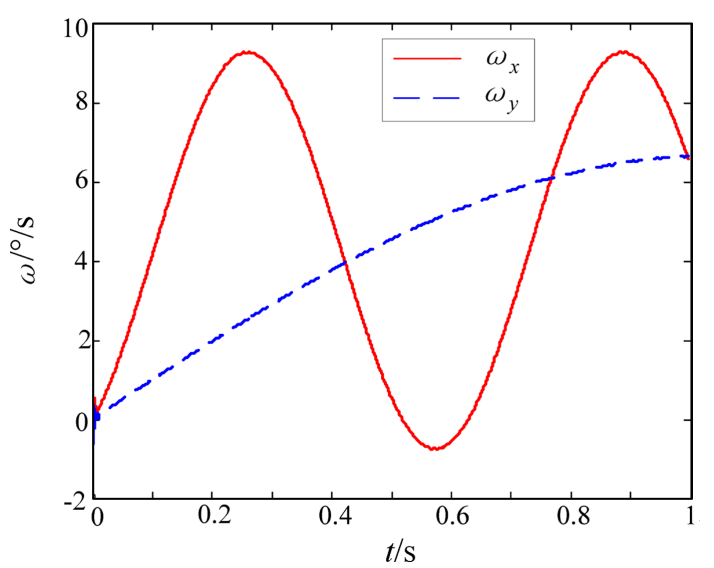

Fig. 10. (Color online) Measurement result of MSSG after compensation.

imbalance. As is shown in Fig. 9, the real (red) and dotted (blue) lines delegate the measured angular velocities of $\omega_{x}$ and $\omega_{y}$ respectively. Clearly, due to the dynamic imbalance, there are corresponding errors superimpose to the true value of the input angular velocity. However, when it comes to the proposed compensation method, the changing curves of the tilted angles are satisfactory, as in shown in Fig. 10.

Figure 11 and Fig. 12 reveal the effectiveness of the compensation method, the measurement errors in $x$ - and $y$-direction are compared before and after compensation. In the figures, the real (red) line delegates the measurement error of $\omega$ before compensation, and the dotted (black) line represents the measurement error of $\omega$ after compensation. According to Fig. 11 and Fig. 12, the measurement errors in $x$ - and $y$ - direction introduced by the dynamic imbalance is synchronized with the rotor rotation speed $\Omega$ and their amplitudes are nearly $0.62^{\circ} / \mathrm{s}$. As for the proposed method, the peak values of measurement errors have been reduced from $0.62 \%$ to $0.03 \%$,

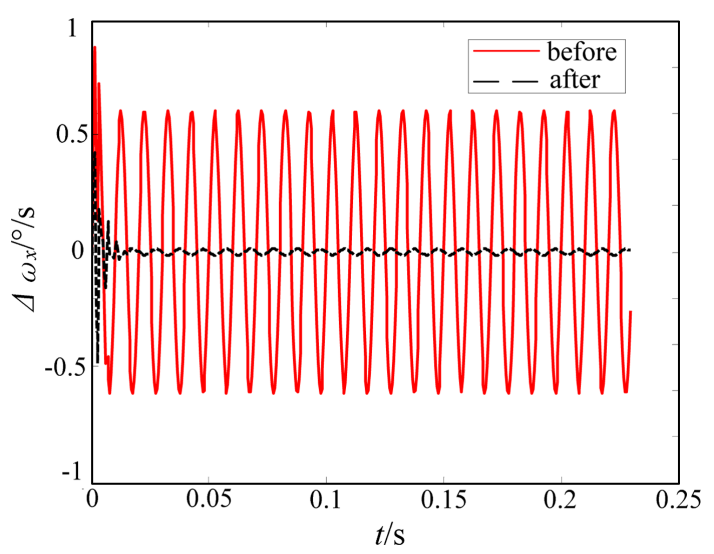

Fig. 11. (Color online) Measurement errors in $x$ - direction. 


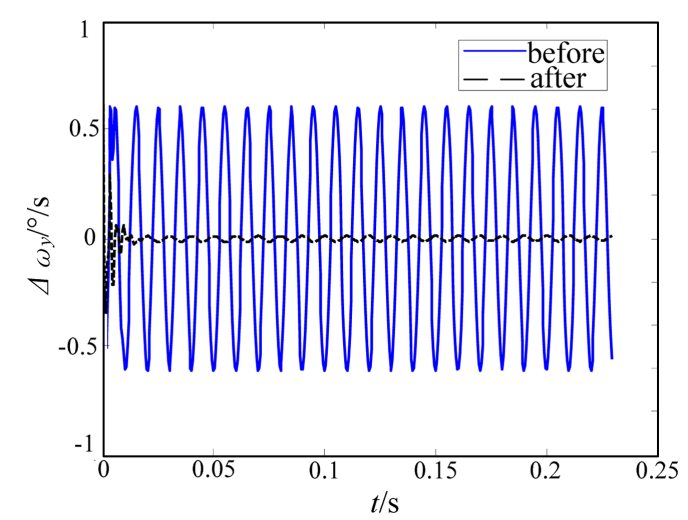

Fig. 12. (Color online) Measurement errors in $y$ - direction.

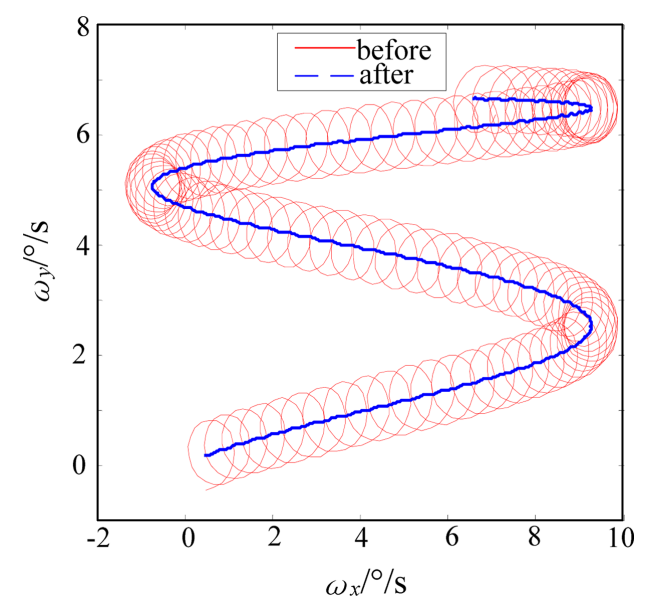

Fig. 13. (Color online) Orbit of the measurement results with an attitude angular rate input.

approximately $95 \%$ in $0.01 \mathrm{~s}$. It demonstrates that, compared with the result without compensation, the measurement errors produced by the dynamic imbalance have been greatly compensated by the proposed method.

The orbits of the geometric axis angular velocity corresponding to Fig. 9 and Fig. 10 are shown in Fig. 13. Also the real (red) and dotted (blue) lines represent the original orbit and the compensated orbit of the angular velocities. The results confirm the point that the dynamic imbalance error has been greatly compensated by the proposed method. And the angular velocity errors produced by the dynamic imbalance in both the $x$ - and $y$-axis have been greatly reduced. And the simulation result is in accordance with the analysis in Section 3.

\section{Conclusion}

To compensate the measurement error caused by dynamic imbalance in a newly developed MSSG, a novel compensation method based on analysis and modeling of the dynamic imbalance error is proposed in this paper. Analytical models of the dynamic imbalance error have been founded, and principle of the compensation method is presented in detail. Simulations results demonstrate that with the proposed compensation method the measurement error can be reduced approximately $95 \%$ in $0.01 \mathrm{~s}$, so that increase the measurement accuracy of angular velocity for a MSSG. To sum up, the presented method can effectively compensate the measurement error caused by dynamic imbalance of the MSSG without adding accessory apparatus, realizing its high-precision attitude measurement. The same compensation method can be extended to other configurations with levitating rotors. Future works will extend this research and build an error identification subsystem to compensate both static mass imbalance and dynamic imbalance errors online based on MSSGs simultaneously. What is presented in this paper may serve as a basis for further research.

\section{References}

[1] J. Q. Tang, B. Liu, J. C. Fang, and S. S. Ge, J. Vib. Control 19, 1962 (2013).

[2] Y. Ren and J. C. Fang. Math. Probl. Eng. 2014, 1 (2014).

[3] X. C. Chen, Y. Ren, and J. C. Fang, J. Mech. Eng. Sci. 228, 2303 (2014).

[4] Y. Ren and J. C. Fang, IEEE Trans. Ind. Electron. 61, 1539 (2014).

[5] C. J. Xin, Y. W. Cai, Y. Ren, and Y. H. Fan, J. Magn. 21, 356 (2016).

[6] Y. Maruyama, T. Mizuno, M. Takasaki, Y. Ishino, and H. Kameno, J. Mechatronics 19, 1261 (2009).

[7] Y. Maruyama, T. Mizuno, M. Takasaki, Y. Ishino, and H. Kameno, J. Sys. Design Dyna. 3, 954 (2009).

[8] J. F. Shortle and M. B. Mendel, Probabilist Eng. Mech. 11, 31 (1996).

[9] C. J. Xin, Y. W. Cai, and Y. Ren, J. Mech. Eng. Sci. doi: 10.1177/0954406216629503(2016).

[10] Y. Maruyama, T. Mizuno, M. Takasaki, Y. Ishino, H. Kameno, and A. Kubo, IEEE Trans Ind Electron. 61, 1911 (2009).

[11] H. Raoul, B. Philipp, and G. Conrad. IEEE Trans Control Syst. and Technol. 4, 580 (1996).

[12] S. Jue, R. Zmood, and L. Qin. Control Eng. Pract. 12, 283 (2004).

[13] A. Tomohiro, T. Mizuno, T. Masaya, and Y. Ishino, J. Jpn. Soc. Appl. Electromagn. Mech. 22, 220 (2014).

[14] T. Mizuno, A. Tomohiro, T. Masaya, and Y. Ishino, IEEE/ASME Trans Mechatronics 21, 1151 (2016).

[15] T. Schuhmann, W. Hofmann, and R. Werner, IEEE Trans Ind. Electron. 59, 821 (2012).

[16] Y. H. Fan, J. Y. Chen, D. L. Weng, and Y. T. Lee, J. Appl. Phys. 103, 103 (2008).

[17] M. Queiroz, J. Vib. Control. 15, 1365 (2009).

[18] P. L. Cui, J. X. He, J. C. Fang, X. B. Xu, J. Cui, and S. Yang, J. Vib. Control. doi: 10.1177/1077546315576430 (2015). 